\title{
Erratum to: Studies on the therapeutic effect of propolis along with standard antibacterial drug in Salmonella enterica serovar Typhimurium infected BALB/C mice
}

Preeti Kalia ${ }^{1 *}$, Neelima R. Kumar ${ }^{1}$ and Kusum Harjai ${ }^{2}$

\section{Erratum}

Following publication of the original article [1] it was brought to our attention that some incorrect superscripts had been added to the three bars for group CP4 in Fig. 1. Please therefore see below for the corrected figure:

Please note that the original article was corrected on the BioMed Central website.

\footnotetext{
Author details

'Department of Zoology, Panjab University, Chandigarh, India. ${ }^{2}$ Department

of Microbiology, Panjab University, Chandigarh, India.
}

Received: 16 December 2016 Accepted: 16 December 2016

Published online: 05 January 2017

\section{Reference}

1. Kalia, et al. Studies on the therapeutic effect of propolis along with standard antibacterial drug in Salmonella enterica serovar Typhimurium infected BALB/c mice. BMC Complementary and Alternative Medicine. 2016;16:485. 


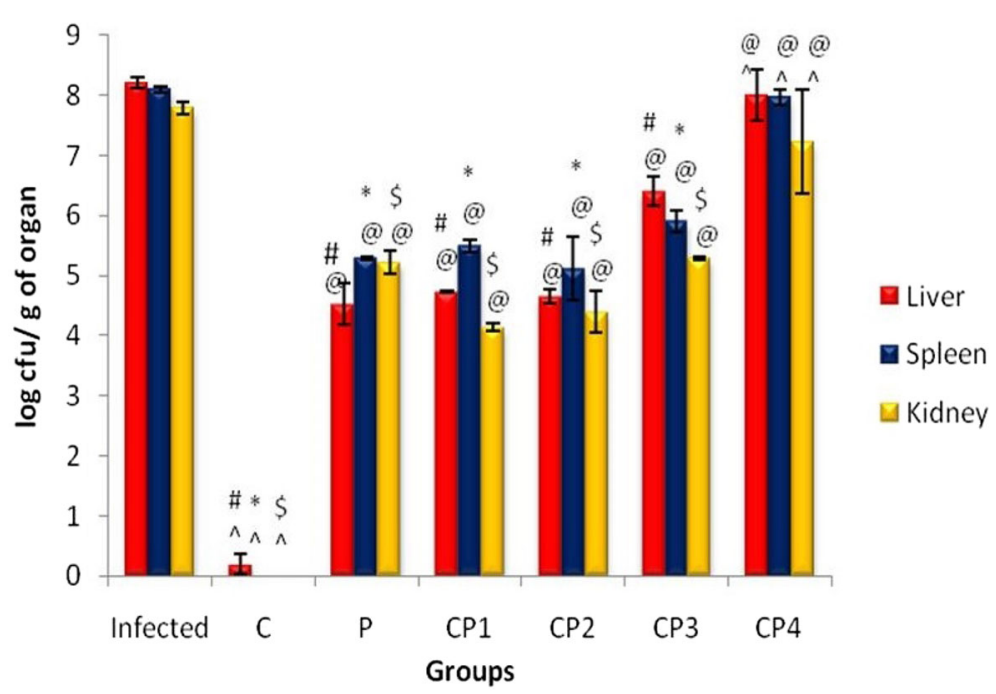

Fig. 1 Histogram showing the bacterial load in different organs of mice after Salmonella enterica serovar Typhimurium infection and treatment in combination groups. Data is expressed as mean $\pm \mathrm{SD}$. ${ }^{*} p$-value Infected liver vs treated liver, ${ }^{*} p$-value Infected spleen vs treated spleen. ${ }^{\$} p$-value Infected kidney vs treated kidney. ${ }^{@} p$-value C vs P, CP1, CP2, CP3 and CP4. ^p-value P vs C, CP1, CP2, CP3 and CP4. ( $\left.{ }^{@} \wedge p<0.05\right)$, $(\# * \$ p<0.001)$ 